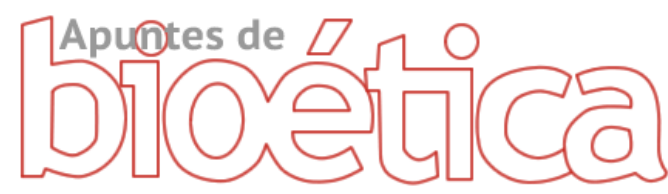

https://doi.org/10.35383/apuntes.v3i1.387

\title{
La tánato - ética, una disciplina necesaria para tiempos de muerte
}

\author{
Farfán Zamalloa P. ${ }^{1}$
}

\section{INFORMACIÓN DEL ARTÍCULO RESUMEN}

Historia del artículo:

Recibido el 08 de mayo 2020

Aceptado el 23 de julio 2020

\section{Palabras claves: \\ Muerte \\ Ética \\ Tánato - ética \\ Filosofía}

La muerte es una realidad que acompaña al ser humano desde sus inicios. Esta característica de finitud ha llevado a que el hombre se pregunte sobre si tiene valor o no la muerte y qué implicancias tiene para su existencia. Desde antiguo, la filosofía ha reflexionado en torno al sentido que aporta la muerte; con el paso del tiempo la bioética ha hecho lo mismo. Sin embargo, es necesario ahora reflexionar sobre la muerte a la luz de una disciplina relativamente nueva, llamada tánato - ética. Por ello, se reflexionó sobre lo que significa hoy la realidad de la muerte. Tal realidad es muy compleja, por tanto, nos llevó a tener en cuenta distintos saberes tanto teóricos en los que se consideró la búsqueda filosófica y las implicancias éticas de la realidad mortal del ser humano que rodean el hecho del morir; y otros, prácticos, en los que se hizo análisis de las dimensiones que rodean al hecho concreto de morir. Posteriormente, se analizó la implicancia de la eutanasia y el suicidio asistido y finalmente, se concluyó dando a conocer la importancia de esta disciplina en la actualidad.

\section{Thanato - ethics, a necessary discipline for times of death}

\section{ABSTRACT}

Keywords:

Death

Ethics

Thanato-Ethics

Philosophy
Death accompanies life from the very moment of conception. This finite character of the human being has led people to wonder about whether death has value or not, and what are the implications of this question for their existence. From time immemorial, philosophy has reflected on the meaning of death; Bioethics has done the same over time. However, now the reflection on the meaning of death can be accomplished in light of a relatively new discipline, called Thanato-Ethics. Hence, this article explores, through this new discipline, the question of what the meaning of death is. Since death is a very complex reality, we had to take into consideration theoretic fields of knowledge -philosophy and the ethics of death- and practical knowledge -such as an analysis of the dimensions that surround the concrete act of dying. Later, we analyze the implication of euthanasia and assisted suicide. Finally, we conclude recognizing the importance and relevance of this discipline today.

\footnotetext{
1 Maestro en Persona Matrimonio y Familia, Teólogo por la Pontificia Universidad Javeriana, Bogotá, Colombia. Email: troper7 2@hotmail.com ORCID: https://orcid.org/0000-0002-5213-5535
} 


\section{Introducción}

El tema de la muerte está presente en la vida desde la concepción, y es por ello que son muchas ramas de las ciencias sociales y del ámbito religioso que la reflexionan continuamente y hay muchas perspectivas para analizarla.

Algunos estudios nos llevan a ver las distintas formas en el cómo asume la persona su finitud. Ramos (2017) afirma: "El modo en el que hemos querido ir comprendiendo este proceso de final de la vida ha sufrido muchas modificaciones, poniéndose el acento en posturas que aceptan la muerte, mientras que otras parecen intentar evadirla" (p.20). Lo que nos muestra la forma en que este hecho natural por la que la humanidad tendrá que pasar tarde o temprano es asumido según la formación y experiencia de las mismas personas.

Así mismo, en la actualidad, "ese proceso se enmarca en un espacio hospitalario, tecnificado y con grandes dispositivos a su alrededor. No obstante, da la sensación de que volvemos a recoger algunos enfoques tradicionales en torno a la propia muerte" (Ramos, 2017 p.20). De allí que debemos ser conscientes que la muerte es una realidad inminente que por más que queramos prolongarla estaremos seguros de que alcanzará a todos en algún momento. Sin embargo, no podemos olvidar que, en todo el proceso vinculado a la muerte, la persona quiere participar de forma activa en el desenlace, pretende introducir a la familia y anhela ser acompañada por los profesionales sanitarios (Ramos, 2017). De allí se va a ir perfilando que la disciplina de la tánato - ética nos tiene que llevar a buscar una mirada más centrada en la persona $y$ en su dignidad y en todo aquello que lo rodea en torno a la muerte como son sus valores, creencias etc.
Sin embargo, la situación actual del SARS Coronavirus 2, el que el Perú y el mundo se ha visto ante una nueva realidad, que no solo ha cambiado drásticamente el estilo de vida, sino que también ha dado un giro total a la humanización de la muerte que buscaba naturalmente el hombre.

Ante esta pandemia, se ha visto en el mundo personas muriendo en las calles, siendo recogidas, en algunos casos, días posteriores, por personal sanitario cuyo protocolo es el de incinerarlos o enterrarlos en lugares alejados, sin la presencia de familiar alguno. Así mismo, una vez que un paciente ingresa a un nosocomio por esta enfermedad y lo lleva a la muerte, los familiares pierden contacto total con el paciente desde su ingreso al centro sanitario, por normas de salud pública.

De allí que necesitemos recordar que después de las dos guerras mundiales, la humanidad no había experimentado una tasa de mortalidad tan elevada, y mucho menos que nos lleve a pensar la realidad de la muerte de otra manera o por lo menos a enfrentarla con vivencias un tanto particulares; ni tampoco "una enfermedad, que, dentro de ciertos límites aceptados por las ciencias epidemiológicas, no siguiera su curso natural, con pocas posibilidades de tratamiento o el desarrollo rápido una vacuna que contuviera su veloz propagación" (Salgado, L; De la Ossa V; 2020, p. 12).

A esto se le suma, la deshumanización de la muerte que hemos descrito antes y las posibles consecuencias que esta nueva realidad, pudiera acarrear en los años posteriores, en los que existirán familiares que no pudieron acompañar a sus muertos ni tendrán un lugar para ir a visitarlos, como es costumbre arraigada en nuestra población. 
Aproximación a la Tánato - ética

Para aproximarnos a una definición y a su campo de estudio tenemos que recordar que estamos frente a un concepto relativamente nuevo, sin embargo, hay que reconocer que, desde la antigüedad, de un modo especial los griegos fueron los que hicieron ya una reflexión en torno a la muerte. Hoy en día, ya se está concibiendo como una disciplina autónoma. Así mismo, no se puede negar que muchos de los aspectos de esta, han sido hasta ahora reflexionados por la bioética.

La realidad de la muerte, que es lo que se encargará de estudiar la tánato - ética, es muy compleja para la misma existencia de la persona pues para su comprensión y estudio se incluyen diversos factores culturales, antropológicos, jurídicos, educativos y psicológicos que deben tenerse en cuenta para su reflexión. Y a la vez, es necesario preguntarse por la misma persona, la familia y su entorno. Todo lo mencionado hace de esta disciplina una tarea compleja de entender, sistematizar y asimilar la realidad de la muerte. Finalmente, se debe recordar la existencia de la tanatología que estudia el campo teórico y práctico de los fenómenos psíquicos, físicos y culturales de la muerte, sin embargo, la parte ética no la toma en cuenta.

\section{Una nueva disciplina}

Es una disciplina nueva pero que contempla la inspiración griega de los conceptos "thanásimos que significa mortal, próximo a morir. Thanatáo expresa el deseo de morir. Thánatos que viene a ser la muerte, el homicidio, el asesinato, y en un sentido figurado, la personificación de la muerte. $\mathrm{Y}$ por último thanatóo que sería matar, dar muerte o mortificar" (Bonete, 2000 p.59). Estos conceptos nos sitúan ante la realidad misma del final de la vida y de los problemas éticos y morales que acarrean el deceso de la persona por lo que se llama esta disciplina tánato - ética.
Como toda ciencia o disciplina tiene su objetivo, el cual sería como dice Bonete: "establecer principios éticos y criterios morales para orientar en las decisiones que se han de tomar en torno al que está próximo a morir por parte del personal sanitario, la familia e incluso el propio enfermo" (p.60); es decir, tiene que ver con la realidad mortal propia del hombre y por ello tendrá que entablar un diálogo con la filosofía para reflexionar en el significado ético de la muerte. Más aún, la tánato - ética tendrá entonces, un doble sentido, uno reflexivo o teórico y otro sentido práctico. Para una mayor comprensión de esta disciplina intentaremos desarrollar, brevemente, qué aspectos se deben cuenta en cada uno de los sentidos mencionados. Creemos que no son los únicos, pero pueden ayudar a comprender dicha disciplina.

\section{En el sentido reflexivo o teórico}

En este primer sentido debemos tener en cuenta algunos aportes de otros saberes que contribuyen a la reflexión teórica de la tánato ética. A saber:

El saber de la filosofía en torno a la muerte:

La filosofía como ciencia a lo largo de la historia, ha reflexionado en torno a la realidad de la muerte. Por ello, es necesario realizar una investigación de los pensadores desde la antigüedad hasta hoy y así, elaborar una síntesis de las diversas filosofías de la muerte. Creemos que sería bueno destacar a Platón, Epicuro, San Agustín, Feuerbach, Heidegger, Levinas, Sartre, entre otros, como es el caso de Schopenhauer que dice "sin la muerte difícilmente se hubiera filosofado" (Shopenhauer, 2007, p. 81).

Así mismo, si queremos pensar bien este aporte filosófico a la tánato - ética debemos plantear algunas preguntas, por ejemplo: a la 
filosofía de la historia, cabe preguntar isi la historia humana tiene un fin, una meta, un sentido cuando la muerte a lo largo de los siglos va desapareciendo civilizaciones? A la antropología filosófica, ¿si vale la pena pensar sobre la identidad personal sin la conciencia de la muerte? En la metafísica, ¿se puede pensar el ser sin la realidad humana de la muerte? En la filosofía de la religión, ¿si son las creencias religiosas las que acentúan la realidad mortal del ser humano o si es la muerte el origen de las creencias religiosas? Y en la filosofía moral, ¿el hombre posee de verdad dignidad o puede ser considerado un fin cuando la muerte nos convierte $y$ hace que nos cosifiquen como cadáveres o restos? (Bonete, 2000).

El saber educativo de la muerte:

Es necesario preguntarse si la concepción de la vida y la muerte, planteada por los filósofos a lo largo de la historia, pueden ayudar a enfrentarnos con espíritu sereno al hecho concreto de la muerte. Parece que esto es verdad si miramos en algunos, como en el caso de Sócrates, que nos presenta Pantón en la obra Fedón donde considera que la práctica de la filosofía en esencia no es otra cosa que ir aprendiendo a morir (Platón 1982). Esto nos lleva a tener en cuenta que la tánato - ética ayudaría a pensar la existencia humana como un modo para erradicar el temor a la muerte y fomentar actitudes morales para asumir la vida con plena conciencia.

También, la investigación de las reflexiones filosóficas nos han de llevar a pensar sobre qué nos enseña la muerte para enfrentarnos a la vida cotidiana, como es el caso de Séneca, que en la meditación sobre la muerte nos ayuda a obtener lecciones morales para la vida de todo hombre, ya que, si quiere adquirir la felicidad, ha de aprender a seguir (Martín, 1984). De esta manera, el saber educativo de la muerte se convierte en una herramienta para asumir la finitud de nuestra vida con serenidad y fortaleza.
El saber ético de la muerte:

Si el saber filosófico nos enseña a concebir la muerte como una realidad que debemos asumirla, cabe entonces verificar si la vida tiene algún sentido a pesar de nuestro ser mortal; por ello, es necesario preguntarnos qué aporta la muerte a la vida moral de la persona. También es necesario no olvidar que nuestra felicidad y nuestros proyectos se ven condicionados por la finitud de la muerte. Por ello, la tánato - ética ha de considerar en su reflexión cuáles son las repercusiones para nuestra vida moral.

No podemos perder de vista que, en cierta medida, la muerte puede elevar el grado de moralidad de las acciones o pueden ser una amenaza a la tensión humana de la búsqueda de la felicidad, ya que, paradójicamente, la muerte concede también el sentido a la vida. En este sentido la tánato- ética reflexiona en torno a cómo poder vivir la felicidad, la libertad y, por ende, el desarrollo de nuestra personalidad moral y los principios éticos incondicionales de nuestra realidad de seres finitos (Bonete, 2000). Es decir, que la muerte como realidad concreta del hombre, puede convertirse en criterio desde el cual valorar nuestras acciones como morales o inmorales. Esto dependerá de que si la muerte le arrebata o al contario le aumenta el grado de moralidad como son los casos de los héroes patrios en que su muerte evidencia una acción moral o el caso de los que se acaban su existencia cometiendo un atentado.

\section{En el sentido práctico}

En este segundo sentido encontraremos algunos saberes, que como ya se dijo, no pretenden ser los únicos, pero que en cierta medida ayudarán hacer de la tánato - ética una disciplina que debe ser comprendida por el ser humano para enfrentar la realidad que nos circunda a diario, que es la misma muerte. 
El saber cultural de la muerte:

Cada cultura expresa de distintas formas la realidad de la muerte, sin embargo, en la cultura occidental, se ha presenciado un cambio en el que no pretendemos detenernos ni hacer un análisis al respecto, simplemente, notaremos lo que resalta y puede ser observado, nos atreveríamos a decir, por todos o una gran mayoría.

Hasta casi finales del siglo XIX, se experimentaba como un acontecimiento familiar y público. Esta situación nos muestra los diferentes cuadros pictóricos, relatos históricos y escenas en las que el moribundo está rodeado de su familia y amigos, en la que pronuncia sus últimas palabras frente a ellos. La muerte en el hogar se producía después de un periodo de enfermedad y dolor; además, el moribundo era consciente de su situación y hasta incluso, si eran creyentes, los invitaban a reconciliarse con Dios (Bonete, 2000).

Toda esta realidad con el paso de los años ha cambiado, como nos dice el historiador Philipe Ariès: desde finales del siglo XIX, una nueva cultura del morir se ha ido imponiendo: se empieza a marginar al moribundo, se le oculta la verdad de su situación terminal, la gravedad de su estado, se le arrincona en el hogar ${ }^{2}$. En esta nueva concepción de asumir la muerte se priva a la sociedad del sufrimiento y la agonía del que está pronto a pasar al umbral de la muerte. Es más, durante el siglo XX y XXI, sobre todo ha ido ganando terreno el morir hospitalario, ya que morir en casa es enojoso y, por lo tanto, la persona ya no muere junto a su familia en la intimidad del hogar, sino que rodeado de aparatos extraños a la misma persona (como son hoy en día los respiradores artificiales) y muchas veces, solo. Esto nos hace ver que según la cultura tanatológica no son mortales y que mueren accidentalmente en los hospitales o por un fracaso de los profesionales de la salud.

Hoy parece que hemos llegado a una mentalidad utilitarista de la muerte donde ya no es un acontecimiento humano y espiritual sino técnico y orgánico que lleva a que el moribundo pierda su ser propio, su identidad, su conciencia, su relación con el mundo y sus seres queridos y acabe en una muerte solitaria y silenciosa en un nosocomio; es decir, la muerte ha perdido incluso su sentido trascendente y el valor de la compañía familiar.

No podemos olvidar, que, en la actualidad, hay una tendencia cultural un poco más humanitaria que ha ido surgiendo, como son los hospitales de cuidados paliativos y las casas de acogida, facilitando así que los enfermos terminales sean tratados por especialistas y que pasen sus últimos días en compañía de los suyos. Al respecto del saber cultural que la tánato ética debe considerar, existen varios autores como: Kessler; Burdin, Suárez ${ }^{3}$, entre otros. A todos ellos se les debe considerar al momento de pensar, desde la cultura, la realidad de la muerte.

\section{El saber comunicativo de la muerte:}

Llamamos saber comunicativo de la muerte al derecho que tiene toda persona a ser informada de su próxima partida inminente. Hoy nos encontramos con una doble realidad acerca de este saber, pues por un lado está quienes quieren negar a toda costa que la persona, sobre todo en estado terminal, se entere que ya se acerca su final y por otro lado, está quienes tienen el deseo y lo comunican a sus seres queridos, por más que esto signifique que tengan que atravesar por un dolor más al que ya vive la familia.

${ }^{2}$ Este es un tema en el que podemos profundizar en ARIÈS Ph. En su libro llamado El hombre ante la muerte.

${ }^{3}$ KESSLER, D, El derecho de morir en paz y con dignidad; BURDIN, I, Decir la muerte, palabras para vivirla; SUÁREZ, E, Cuando la muerte se acerca. 
La tánato - ética tendrá que beber de este saber, al mismo tiempo que deberá reflexionar con argumentos morales y éticos, sobre si vale la pena informar con la verdad al enfermo terminal $y$, si es afirmativa la respuesta, deberá discernir sobre quién y cómo se ha de comunicar a la persona su pronta su partida.

Algunos ya han reflexionado sobre esta realidad y llegan a la conclusión que la persona tiene derecho a saber la verdad de su enfermedad y la verdad de su pronto deceso. Además, están de acuerdo en que el que comunique no sea el médico o una persona externa a su círculo cercano, es decir que la familia es quien debe transmitir al enfermo la verdadera situación para ayudarle a concientizar y entrar en el misterio de muerte (Cabodevilla, 2001). Así mismo, creemos que esta disciplina nos debe ayudar a vivir los últimos momentos, como privilegiados para tomar conciencia de la existencia del ser humano y para aceptar, de manera madura y digna, la muerte propia.

El saber deontológico de la muerte:

Este campo es muy complejo y no nos detendremos, pero sí debe ser un eje funadamental en la tánato - ética, sobre todo, con el tema de la eutanasia. Por ello, debe reflexionar y aclarar con precisión los conceptos de eutanasia, distanasia, ortotanasia, etc. Sin embargo, la dimensión deontológica es sustancial y a veces olvidada, sobre todo cuando se refiere al problema moral que se da hoy en día, de acuerdo con las posiciones que toman las sociedades y gobiernos. Este dilema, que sobre todo los profesionales de la salud y de un modo especial los médicos, son los que deben tener una mayor formación y claridad al respecto, ya que las mismas familias y la sociedad los hacen los primeros responsables frente a los pacientes y las decisiones que se toman.
El Saber técnico del morir:

Estamos frente a sociedades que, en su gran mayoría, buscan prolongar la vida de un enfermo terminal a través de recursos técnicos que han ido suscitando problemas morales de diferente índole. La tánato - ética tiene, tomando esta realidad en cuenta, que dar una respuesta ante la pregunta: ¿se ha de mantener artificialmente la vida de una persona? Pero la dará teniendo en cuenta no solo el uso de los métodos ordinarios y extraordinarios o medios proporcionados o desproporcionados que existen, sino que, el debate se centre más en la situación real del enfermo, en función a sus expectativas reales de curación, el proceso de su enfermedad, las implicaciones sociales y familiares derivadas de estas situaciones, que muchas veces son límites.

El saber clínico de la muerte:

La tánato - ética deberá tener en cuenta este saber, en cuanto que el diagnóstico sobre cuando uno está muerto se ha convertido en un problema moral y con argumentos en contra y a favor cuando existe lo que es llamada la muerte cerebral ${ }^{4}$. Más aún, le incumbe este problema por las repercusiones morales que conlleva la definición clínica de la muerte y por las preguntas que se hacen los expertos médicos y científicos tales como icuándo estamos realmente muertos? ¿cuándo o no se pueden extraer órganos de los pacientes clínicamente muertos? ¿o qué debemos hacer con los cadáveres? Estas preguntas deberán ser contestadas por esta disciplina, contando con las referencias éticas y médicas claras, puesto que, de lo contrario, se corre el riesgo de torturar a una persona todavía no muerta.

Como mencionamos anteriormente, estos no pretenden ser los únicos saberes que la

\footnotetext{
${ }^{4}$ Para poder comprender mejor la discusión sobre la muerte cerebral se pueden revisar autores como ESCALANTE , J, Muerte encefálica, evolución histórica y situación actual; REQUENA, P, El diagnóstico de muerte cerebral.
} 
tánato - ética debe considerar, al momento de hacer su reflexión. A la vez no podemos olvidar como disciplina relativamente nueva que es, que tendrá que ir ampliando los saberes de los cuales se ayudará para dar respuestas éticas y morales cada vez más precisas y en favor de la dignidad del ser humano y de la vida, que ayuden a aceptar la realidad misma de la muerte que nos circunda.

\section{La tánato - ética al final de la vida}

Si bien es cierto más arriba tocamos la necesidad del saber deontológico, aquí queremos hacer brevemente una mirada a la situación actual que estamos viviendo de la pandemia por el COVID-19. Creo que es bueno acércanos a nuestra disciplina, a partir de una mirada práctica y desde la reflexión de la realidad a la que se están enfrentando, sobre todo, los profesionales de la salud. En este sentido, mencionaremos brevemente dos realidades con la que se está lidiando: la eutanasia y el suicidio asistido. No se pretende profundizar sino solo sacar a la luz una reflexión en la que la tánato - ética debe dar una palabra.

Durante muchos años, el ser humano se ha planteado la interrogante sobre el significado del buen morir y para responder esta pregunta, se han propuesto dos opciones que buscan mitigar el dolor que puede padecer una persona a causa de una enfermedad que lo vuelve vulnerable durante su proceso de morir, sin perder el horizonte que el efecto deseado no es acortar ni alargar la vida, evitando la muerte; la misma que nos hace recordar que somos seres finitos, débiles y frágiles.

De lo anterior se desprenden dos términos muy usados en el gremio de la medicina, que han sido el motivo de algunas discusiones éticas, religiosas y políticas en diferentes países del mundo.
El primero hace referencia a la eutanasia entendiéndose como la "conducta (acción u omisión), intencionalmente dirigida, a terminar con la vida de una persona que tiene una enfermedad grave e irreversible, por razones compasivas (esto es, con el fin de minimizar el sufrimiento) y en un contexto médico" (Sociedad Española de Cuidados Paliativos [SECPAL], 2002). En este caso la eutanasia puede realizarse con o sin el consentimiento del individuo.

Por otra parte, encontramos el suicidio medicamente asistido, entendido como la acción que lleva a cabo una persona para terminar con su propia vida, sirviéndose de los medios que le ha proporcionado un médico. En este caso, el suicida lo puede llevar a cabo sin contar con el auxilio de nadie.

Estos dos términos pueden parecer similares, pero difieren en la forma de ver al individuo. En el primer caso, como un agente pasivo que necesita de un agente activo (la mayoría de las veces, un médico), y en el segundo caso, el propio paciente es el que activa el mecanismo para terminar con su vida.

Sin embargo, a pesar de tener claro estos dos conceptos, con el tiempo se han generado nuevas dudas basadas en el deseo de acompañar al que está muriendo, reconociendo lo que significa para el que lo vive, pero también para el cuidador o el acompañante. Debemos reconocer que durante este proceso se genera miedo e incertidumbre, pero también es un tiempo para brindar amor, cuidados, gratitud, y puede ser una instancia de reconciliación, responsabilidad, aprendizaje y transformación para los involucrados.

Partiendo de esto, surge una nueva visión y oportunidad que no solo busca aliviar una enfermedad, sino ayudar a sobrellevar el sufrimiento y el dolor, el cual queremos erradicar de nuestras vidas. Los cuidados paliativos inspirados en las intuiciones de la británica Cicely Sanders, donde se propone una atención integral 
que incluye medicación, tratamiento del síntoma, cuidados diarios, equipamiento, apoyo psicológico, social y espiritual al paciente y a sus familiares, teniendo como principio mejorar la calidad de vida, en lo posible, en pacientes cuya enfermedad no se puede curar, tratando de garantizar un morir sin dolor, morir junto a la familia, en el lugar que se elija, recibir sedación cuando sea necesario y no ser objeto de una obstinación terapéutica 5 .

Teniendo claras las definiciones ya planteadas, surgen nuevas interrogantes que involucran de manera particular al personal médico sobre la pertinencia de legalizar la eutanasia, partiendo del cuestionamiento del objetivo real que se busca cuando se realiza este tipo de prácticas; impedir alargar el dolor y el sufrimiento. Pero la eutanasia ¿es el único medio para evitarlo? Sin duda, con la eutanasia desaparece el sufrimiento, pero al precio de eliminar también al que sufre. Esto nos permite reflexionar y hacer un paralelo, teniendo como base el juramento Hipocrático que profesan los médicos el día que aceptan esta valiosa profesión, llevándonos a la conclusión que debemos buscar nuevas alternativas que nos permitan eliminar el dolor y el sufrimiento, pero no a la persona que lo padece, y este es uno de los objetivos de los cuidados paliativos.

No debemos olvidar algunos principios fundamentales de la práctica médica diaria que nos ayudarán a entender, y a tomar las mejores decisiones frente a nuestros pacientes, uno de ellos es recordar que "el dolor es abstracto, no existe; existen los dolientes, los cuales piden ser valorados, consolados, escuchados, acompañados, y tener seguridad, ante la incertidumbre de la muerte, de ser tratados y cuidados, profesionalmente" (Zurriaráin, 2019, p.184).

\footnotetext{
5 La "obstinación terapéutica" es rechazada por ser contraria a la dignidad de la persona. Sin embargo, sabemos que la medicina contemporánea está constantemente sometida a la tentación de considerar que es éticamente exigible todo lo que es técnicamente posible.
}

Un buen acompañamiento del enfermo, que evita que se sienta abandonado, como un estorbo o como un ser molesto, y un correcto discernimiento de las diferentes situaciones, son parte indispensable para evitar el riesgo del ensañamiento "terapéutico" y de la eutanasia.

Es por eso, que podemos considerar que los cuidados paliativos son la mejor manera de ayudar a morir al enfermo, no "ayudándolo" a terminar con él como dice Zurriaráin (2019) "los cuidados paleativos no tienen como objetivo la muerte del enfermo, sino que cuidan de su vida mientras este llega a su fin, alivian su sufrimiento y proporcionan los medios para una muerte tolerable" (p.186). Por lo que es indispensable que se garantice en todos los sistemas de salud el derecho a tener la oportunidad de incluir el cuidado de la vida del paciente que llega a su fin, en un contexto humanizado, que comprometa una estrecha cooperación de equipos multidisciplinares que promuevan el compromiso y la solidaridad con la salud humana (Zurriaráin 2019). De ahí que la medicina paliativa sea la verdadera solución ética ante la situación terminal de un paciente; pues esta, ayuda a comprender nuestra común vulnerabilidad y nuestra recíproca interdependencia, por la cual podemos sostenernos mutuamente tanto a un buen vivir y a un buen morir (Fascioli, 2016).

Por esta razón, vale la pena pensar sobre la utilidad de una adecuada educación para que el proceso de morir tenga en cuenta los factores culturales, espirituales y emocionales con los que muchas personas afrontan la vida y la muerte; para que la muerte deje de ser un tabú social, y para que los enfermos terminales dejen de ser los grandes olvidados de la medicina y de la comunidad social. 


\section{Conclusiones}

Importancia de la tánato - ética hoy:

- La muerte que está presente en el ser humano desde la concepción y que por lo tanto es una realidad inminente que por más que queramos prolongarla tenemos la certeza que nos llegará a todos en algún momento, nos lleva a reflexionar sobre la importancia de la tánato - ética hoy. Creemos que esta disciplina es importante en nuestro contexto por las siguientes razones:

- La muerte es una constante que acompaña la historia de la humanidad $y$, por ende, un acontecimiento por el que debe pasar toda persona, sociedad o cultura pues nadie escapa de la finitud propia del del ser humano, aunque hoy se encuentren instituciones, avances científicos, etc. que quieran prolongar la vida, usando distintos medios.

- La tánato - ética toca una realidad propia del ser humano a la que muchos temen acercarse, asumirla o aceptarla pero que es necesaria reflexionarla para establecer los principios éticos y criterios morales que orienten las decisiones que se han de tomar en torno al que está próximo a morir.

- Frente a la situación actual de la pandemia del Covid -19, hablar de la dignidad de la persona que está a punto de morir e incluso de tener una muerte digna, lleva a encontrar en esta disciplina luces necesarias que nos ayudan a comprender el hecho de morir desde una ética que englobe el compromiso de la familia, de la persona y del personal sanitario que enfrenta, día a día, la impotencia y las limitaciones que conllevan a tomar decisiones prácticas y para el bien común.
- Es importante hoy, porque no solo se enfoca desde un saber único, sino que se abre a una gama de saberes que fomenta la interdisciplinariedad tan necesaria para la ciencia y para el desarrollo y comprensión del ser humano, rescatando el valor de la persona, la actuación ética, la parte cultural, médica, filosófica y ipor qué no decirlo?, histórica, que ayudan a ver a la muerte no como un fracaso sino como una ayuda necesaria para darle sentido a la existencia humana.

- Es importante porque nos da las herramientas necesarias para los grandes dilemas éticos y morales que circundan alrededor de la muerte y que son de discusión, en la sociedad y en la política misma de un país, como son las orientaciones que se deben seguir en torno a los temas de eutanasia, ensañamiento terapéutico, muerte cerebral, donación de órganos, entre otros.

\section{Bibliografía}

Asociación Española de Bioética y Ética Médica (AEBI) (2018). Comunicado de AEBI sobre la Eutanasia. Morir con dignidad. Madrid (2020): AEBI. Disponible en: Http://aebioetica.org/archivos/Conclusiones_y_comunicado_final.p df. (s. f.).

Benítez, J. (2010). La bioética frente a la muerte, entre el sufrimiento y la eutanasia.PDF. Academia.Ed

https:www.academia.edu/10986978/La_bio \%C3\%A9tica_frente_a_la_muerte_Entre_el_ s ufrimiento_y_laeutanasia 2010. (s. f.).

Bonete, E. (s.f.) Ética de la muerte: de la bio-ética a la tánato-ética. Daimon Revista Internacional De Filosofía, (25), 57-74. Recuperado a partir de https://revistas.um.es/daimon/article/view/1 4801. (S. F.). (s. f.). 
Bonete, E.(s.f.), Implicaciones éticas de la muerte. la ciudad de Dios, R, CCXIII.2000 Pp.1025 -1047. (s. f.).

Bonete, E. (2019). El morir de los sabios. Una mirada ética sobre la muerte, Madrid: Tecnos, 2019. (s. f.).

Cabodevilla, I. (1999). Vivir o morir conscientemente. Desclée de Brouwer, Bilbao.

Escalante, J. (2000) Muerte encefálica. Evolución histórica y situación actual. Revista Medicina Intensiva, Vol. 24, No. 3, 2000. (s. f.).

Escobar, J. (2000). El morir como ejercicio final del derecho a una vida digna. Bogotá, Colombia: Uni- versidad El Bosque; 2000. (s. f.).

Fascioli, A. (2016) los cuidados paliativos al final de la vida: expresión del reconocimiento del otro, Revista, Enfermería: Cuidados Humanizados, Vol. 5, no 2- Diciembre 2016-ISSN: 1688-8375 ISSN en línea: 2393-6606. (s. f.).

Macellari G. (2002) La muerte: Un bien incurable. Desde el arte de morir hasta la eutanasia. Madrid: Coope- ración Editorial; 2002. (s. f.).

Martín, F. El ideal del sabio Séneca, publicación de la Caja de Ahorros de Còrdova, Caps. III y IV, pp. 113 -166. (s. f.).

Méndez, V. (2002) Sobre morir: Eutanasias, derechos, razones. Madrid: Trota; 2002. 02. (s. f.).
Platón, Fedón, Espasa - Calpe, Madrid, 1982. (s. f.).

Requena, P. (2009) El diagnóstico de muerte cerebral, persona y bioética Revista, PP. 128 136. 2009. Julio-Diciembre 2009... (s. f.).

Sánchez, S. (2001) La pasión de morir, preguntas y respuestas desde la vida, Biblioteca Nueva Madrid, (s. f.).

Shopenhauer, A.(s.f.), El amor, las mujeres y la muerte, Edaf; Madrid. (s. f.).

Sociedad española de cuidados paliativos (Secpal). (2002) Declaración sobre la eutanasia. Madrid: Secpal; (s. f.).

Ramos, S. (2017) Algunas reflexiones filosóficas sobre el modo de concebir la muerte, en Revista de Treball Socia (RTS)- no 210, (s. f.).

Salgado, L; De la Ossa V; Montes,D; González T. (2020) Coronavirus, salud pública y mundo Revista Colombiana Ciencia Animal. Recia. 2020; 12(1): e769. Https://doi.org/10.24188/recia.v12.n 1.2020. (s. f.).

Torres, J. (2012), Aspectos éticos y legales de la muerte, Tesina, 24 de abril 2012.Asociación Mexicana de Tanatología. (s. f.).

Zurriaráin, R. (2019), Cuidado paleativos, Soclución ética acorde con la dignidad humana al final de la vida, Revista, Pers Bioet. 2019; 23(2): 180-193. DOI: recuperado a partir de https://doi. Org/10.5294/pebi.2019.23.2.2. (s. f.). 